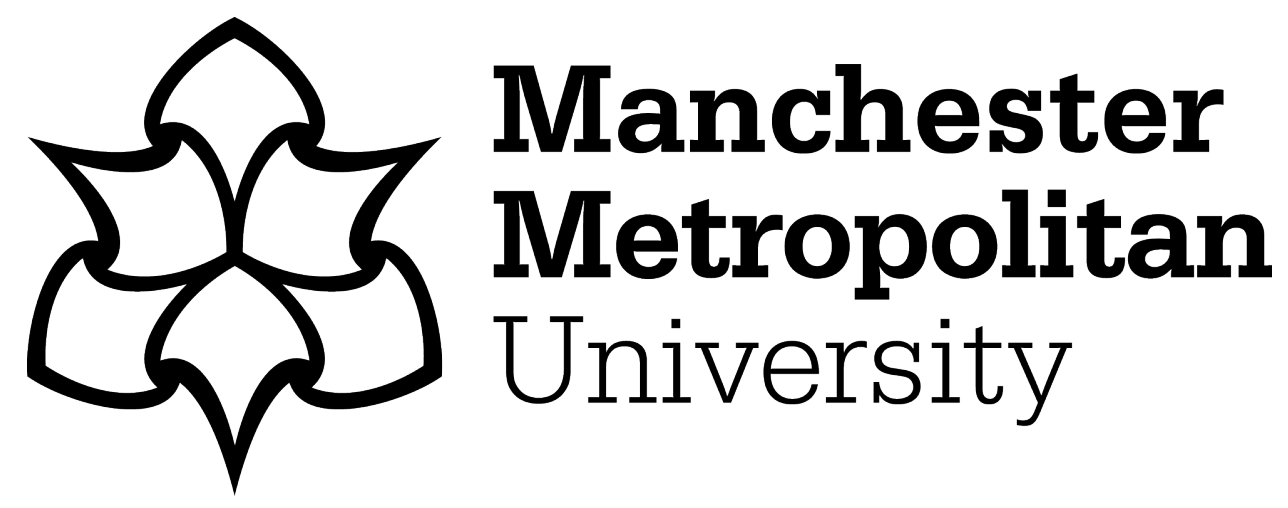

Mei, TX and Li, H (2008) A novel approach for the measurement of absolute train speed. Vehicle System Dynamics, 46 (sup1). pp. 705-715. ISSN 00423114

Downloaded from: https://e-space.mmu.ac.uk/622230/

Publisher: Taylor \& Francis

DOI: https://doi.org/10.1080/00423110802036984

Please cite the published version 


\title{
A novel approach for the measurement of absolute train speed
}

\author{
T.X. Mei* and H. Li \\ The University of Leeds, School of Electronic and Electrical Engineering, Leeds, LS2 9JT, UK
}

(Received 25 July 2007; final version received 6 March 2008)

\begin{abstract}
This paper presents a new approach for the measurement of the absolute travelling speed of rail vehicles. Unlike the conventional techniques where the vehicle speed is obtained from the measurement of rotational speed of wheelset axles, the proposed method derives the speed indirectly from the time shift between motions of any two wheelsets. It will explore vehicle/bogie dynamic responses to track excitations, and extract particular features of motion at the wheelsets from inertial sensors mounted on the bogie frame. The new technique will be able to provide an accurate measurement of the vehicle ground speed, even when the wheel slip/slide occurs under traction/braking. The performance assessment of the proposed measurement method using a conventional bogie vehicle is provided, including the robustness against the parameter variations in the suspensions and the reduced level of track roughness at low travelling speeds.
\end{abstract}

Keywords: absolute train speed; measurement; time shift

\section{Introduction}

The conventional method for the measurement of train speed is to use encoders that measure the rotational angle and hence speed of wheelset axles [1]. However, the measurement accuracy is not only limited by the type of encoders acceptable for the rail application (for reliability considerations), but also by wheel slip/slide in poor contact conditions where the wheel speed may differ substantially from the travelling speed. Alternative solutions have been investigated. The use of radar sensors to measure speed is not new and has been applied to measure vehicle speed [2], although the installation as well as operation on every commercial train will not be straightforward. In [3], eddy current sensors are used to detect inhomogeneities in the magnetic resistance along the tracks, caused by rail clamps, turnout components and irregularities of the rail. The system consists of two identical differential sensors (working at different excitation frequencies) arranged at a distance in the direction of movement, and the vehicle speed is derived from the time delay between the two sensor outputs. Similar approaches are proposed in $[4,5]$, which detects delays between two optical sensors or even video image signals in a fixed distance. In [6], a patent has been filed which measures the time difference between two

*Corresponding author. Email: t.x.mei@leeds.ac.uk

ISSN 0042-3114 print/ISSN 1744-5159 online

(C) 2008 Taylor \& Francis

DOI: $10.1080 / 00423110802036984$

http://www.informaworld.com 
vibration pulses measured from the two wheelset axles when they pass rail fixtures such as joints and switches, etc.

This study investigates a new method that offers a fast and accurate measurement of train speed from wheelset responses to track irregularities. Only inertial sensors mounted onto a bogie frame will be needed in the measurement system, which is an important advantage of the proposed method as it avoids the much harsher working environment of the axles and there are obvious benefits from this type of sensors in terms of reliability, costs and installation. The basic idea of the proposed measurement technique was first introduced in [7], but a more detailed study is presented in this paper which includes the assessment of key practical issues such as robustness against the parameter variations in the suspensions, and limitations due to the level and frequency contents of track roughness at very low travelling speeds.

\section{The proposed measurement method}

In the proposed system, the vehicle speed is detected from a time shift between two signals which are derived from the dynamic responses of a railway bogie at the two wheelsets to track excitations. In particular, it explores vehicle responses to the track irregularities rather than the fixtures. The track misalignments always exist and provide a continuous source of (random) excitation to the wheelsets. Although the magnitude of the excitation may be lower than that from the track fixtures and it is more difficult to detect the time shift between two continuous signals (than that between two pulses), the use of the wheelset movements in response to the random input offers a reliable way to measure the vehicle speed accurately and continuously.

Two inertial sensors will be required and mounted onto a bogie frame to measure the bounce and pitch accelerations as illustrated in Figure 1 - this may be replaced by the lateral and yaw accelerations if the plan-view motions and measurements are considered. The use of bogiebased sensors is one of the obvious advantages of the system because the reliability requirement and also cost of the sensors will be much lower compared with the axle-mounted ones.

Figure 2 shows the basic structure of the measurement method. The measured signals will be processed via two filters to produce estimated wheelset movements (i.e. the track irregularities). The filters are designed to obtain the track estimation within a frequency range particularly suitable for deriving the vehicle speed [7]. Because there is no need for a complete and precise estimation of the track inputs, the filters are much simpler and hence easier for practical implementation than a high-order (full state) estimator which has previously been proposed for applications where the estimation of track irregularities together with that of wheelset/bogie states are the primary objective [8].

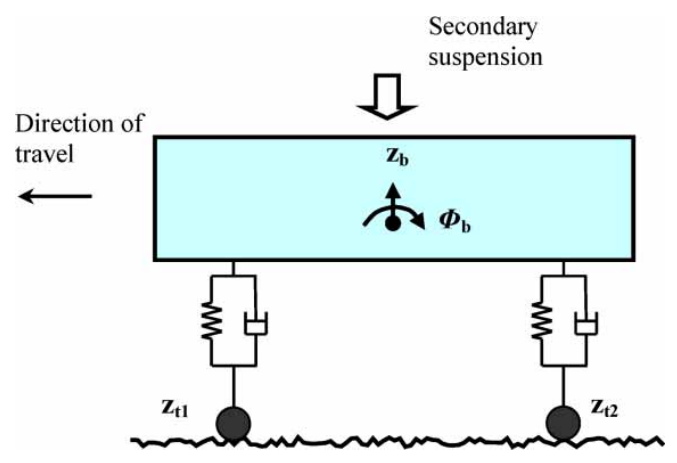

Figure 1. Side view diagram of a conventional railway bogie. 


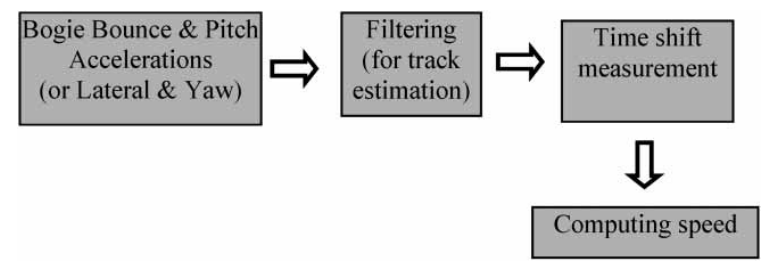

Figure 2. Speed measurement scheme.

The two outputs from the filters represent excitations experienced by the two wheelsets and the time shift between the two is directly determined by the vehicle travelling speed. Therefore the measurement of the time shift is also the measurement of the vehicle speed, as shown in Equation 1.

$$
V_{s}=\frac{2 \cdot L_{\mathrm{b}}}{T_{\text {delay }}}
$$

where $L_{\mathrm{b}}$ is the semi-wheel space of the bogie $(\mathrm{m}), T_{\text {delay }}$ the time shift between the two signals (s) and $V_{\mathrm{s}}$ is the vehicle ground speed $(\mathrm{m} / \mathrm{s})$.

Because the two estimated outputs are used to compute a relative quantity of time, the proposed method is expected to be robust against changes in the system or estimation errors as long as both channels are affected in the similar way, as it will be demonstrated in the next section. Another issue that will be assessed is how much the measurement and the reliability of the measurement will be dependent upon the level as well as the frequency content of track excitations which are potentially problematic at very low speed (and also on very smooth tracks).

\section{Assessment of the fault detection technique}

The side view models of a conventional bogie vehicle are used to demonstrate the effectiveness of the proposed measurement method, which include the bounce and pitch motions of the body frame and the two bogies. The spring and the damper in the primary suspensions are considered as linear components, whereas a linearised airbag model is used to simulate the secondary suspensions.

Two sets of random track data are used in the assessment. A simulated random track (track 1), representing the roughness of a typical main line, is derived to give an appropriate spatial power spectrum $\left(A_{r v} / f_{t}^{2}\right)$ for the track vertical displacement. This is a simplified version of the generalised power spectrum which has higher order terms on the denominator, but for the study the differences are relatively small. A real measured track data for a German high-speed line is also used in the evaluation (track 2).

There are clear differences between the two sets of track data, as demonstrated in Figures 3 and 4 . The high-speed track is of high quality that is necessary to ensure a good level of passenger comfort and therefore the misalignment is significantly lower than the simulated track. The maximum wavelength of the real track data is limited to around $100 \mathrm{~m}$ in this case due to limitations of the measurement tools, whereas the wavelength of the simulated track is clearly much longer. More significantly for the proposed measurement technique, the simulated track is much richer in the low wavelength (high frequency), with a minimum wavelength of about $1 \mathrm{~m}$ (as the data is sampled every $0.5 \mathrm{~m}$ ) compared with around $5 \mathrm{~m}$ for the measured track. 


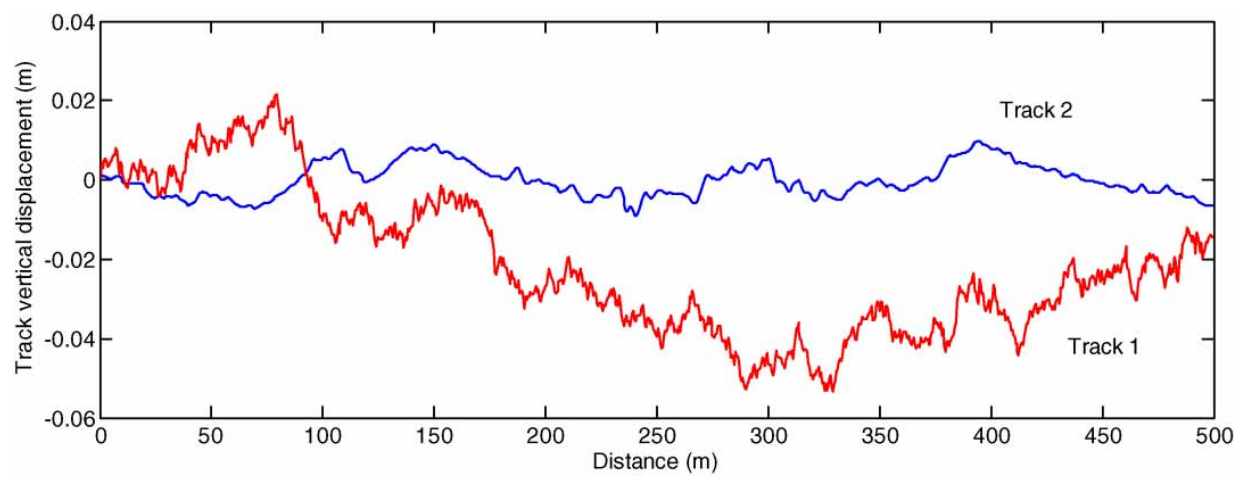

Figure 3. Track irregularities in the vertical direction - displacement.

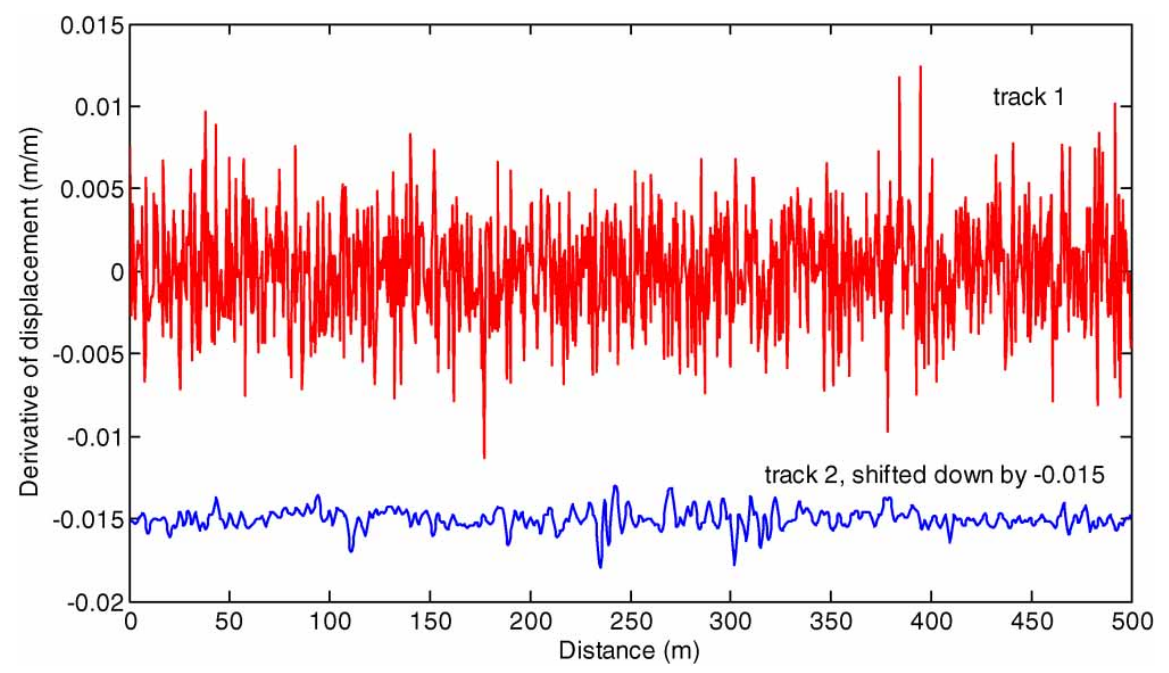

Figure 4. Track irregularities in the vertical direction - derivative of displacement.

The filters are tuned to provide an accurate estimation of the track irregularities for the particular vehicle scheme used in the study. Ideal sensing is initially assumed, and the effect of sensor noises will be discussed later on in the study. Figures 5 and 6 compare the estimated track irregularities with the real inputs used in the computer simulations for the two sets of track data at the vehicle speed of $55 \mathrm{~m} / \mathrm{s}(198 \mathrm{~km} / \mathrm{h})$. A close match in both cases is achieved, although the filters appear to estimate the real measured track more precisely than the simulated one. This is due to the limited bandwidth of the filters, as the estimation errors occur mostly at high frequencies. An increase in the filters' bandwidth would improve the estimation accuracy, but this is not a critical problem for the measurement as the errors will be present to both wheelsets of a bogie and therefore present no major adverse effect on deriving the time shift and hence the speed.

The designed filters work well throughout the speed range. Figure 7 shows a comparison of the estimated track input with the real one (track 1) at a lower speed of $20 \mathrm{~m} / \mathrm{s}(72 \mathrm{~km} / \mathrm{h}$ ) and again a close match is obtained. The results suggest that the use of more sophisticated model-based techniques may not be necessary in the estimation of the track irregularities, as the simpler filters used in the study seem to offer an effective solution. 


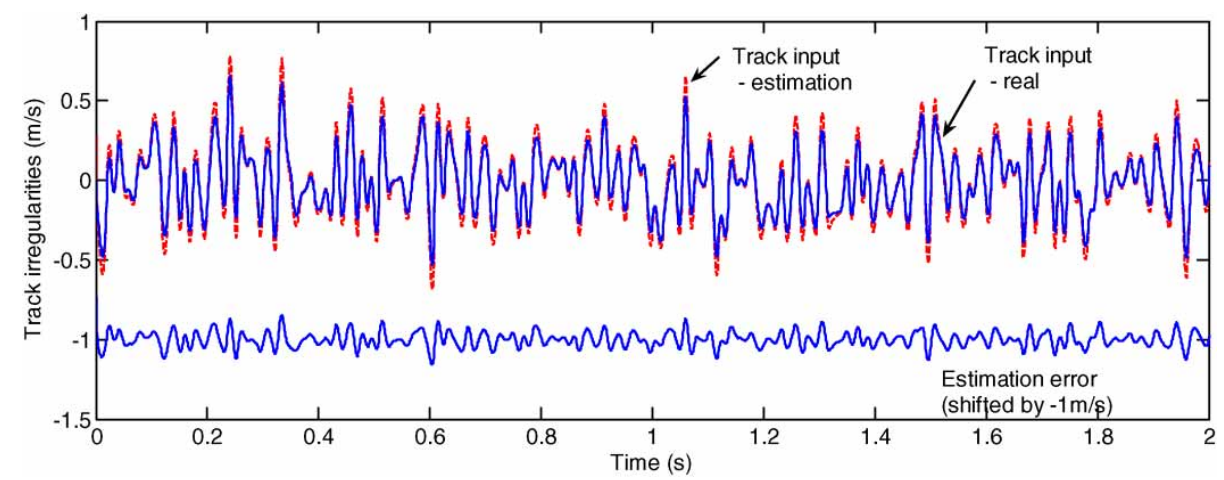

Figure 5. Estimated track irregularities - velocity (track 1, at $55 \mathrm{~m} / \mathrm{s}$ ).

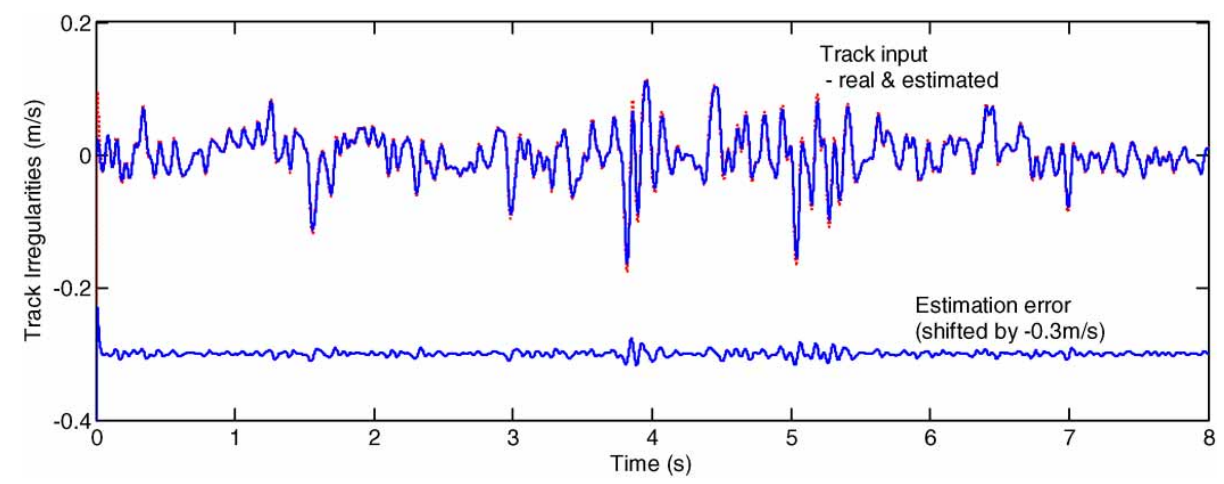

Figure 6. Estimated track irregularities - velocity (track 2, at $55 \mathrm{~m} / \mathrm{s}$ ).

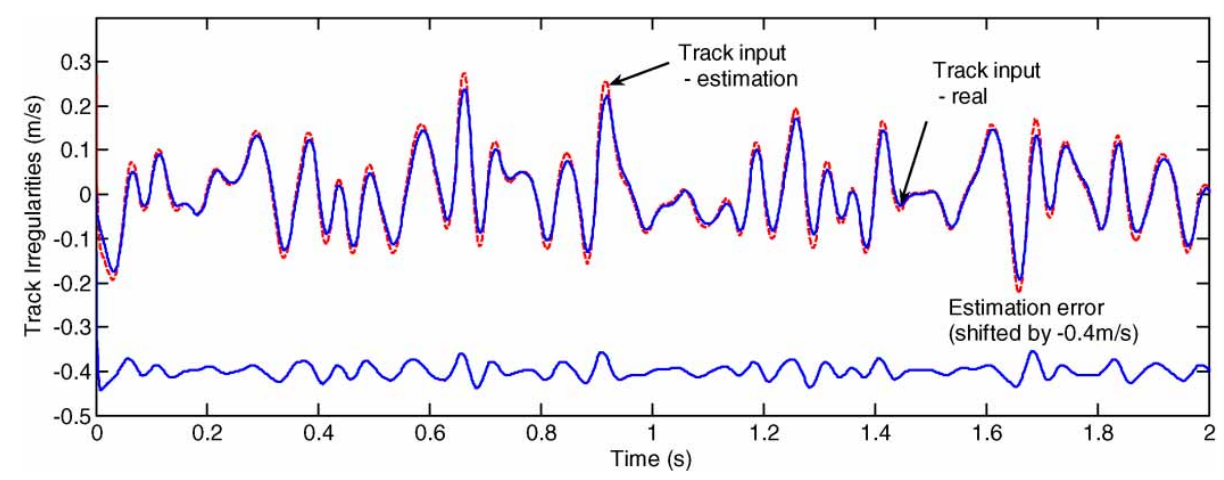

Figure 7. Estimated track irregularities - velocity (track 1, at $20 \mathrm{~m} / \mathrm{s}$ ).

The estimation of the track inputs at the two wheelsets of a bogie enables a reliable detection of the time delay from the leading wheelset to the trailing one. This can be achieved by computing the cross-correlation between the two signals at a fixed sampling rate for a specified time period (the time window). Figure 8 gives the result of the computation for the vehicle speed of $55 \mathrm{~m} / \mathrm{s}$ with the simulated track as input. Two time windows $(0.3$ and $0.5 \mathrm{~s})$ are used. It is obvious that a longer time window gives a more reliable detection of the peak value and the time shift against lower values of data at other times. On the other hand, a longer time may 


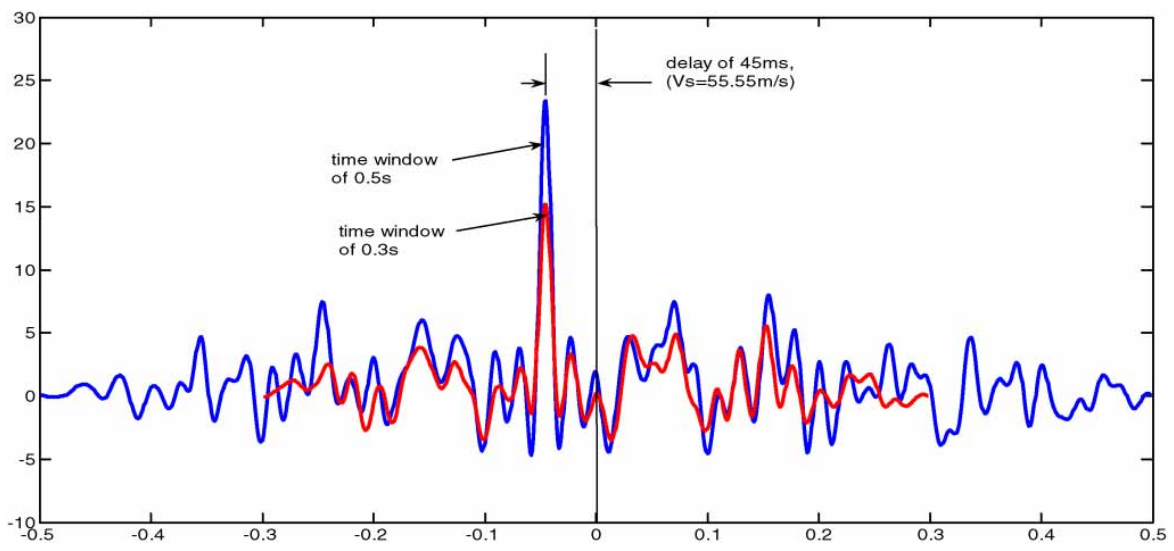

Figure 8. Cross-correlation of estimated track inputs (track 1, at $55 \mathrm{~m} / \mathrm{s}$ ).

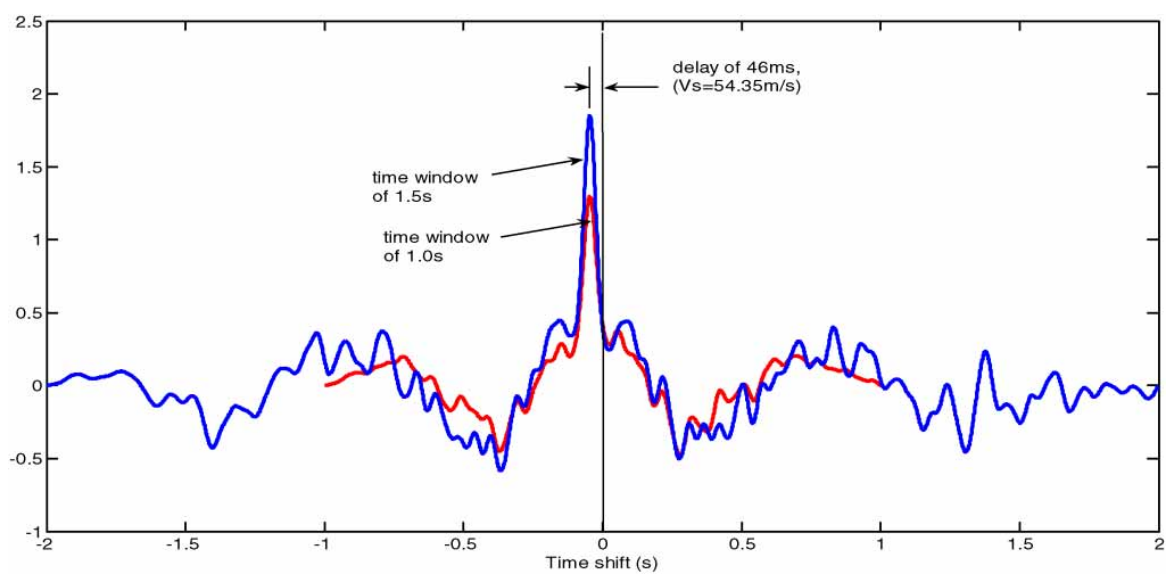

Figure 9. Cross-correlation of estimated track inputs (track 2, at $55 \mathrm{~m} / \mathrm{s}$ ).

cause undesirable delays in updating the measurement output which would be problematic in rapid acceleration or deceleration operations and therefore a suitable balance must be found. The measurement accuracy is directly affected by the time interval used to sample/process the data. The semi-wheel space of the vehicle scheme used in the study is $1.25 \mathrm{~m}$, so a precise time shift should be $2.5 / 55(=0.04545 \ldots)$. However, the sampling rate used in the simulation is $1 \mathrm{~ms}$ and the detected delay is $45 \mathrm{~ms}$ with a truncation error of $0.45 \mathrm{~ms}$ (or $0.45 \mathrm{~m} / \mathrm{s}$ ) resulting in a measurement error of about $1 \%$. This is more of an issue at high speeds where the time shifts are small, but can be improved by reducing the sampling rate. The demand on computation to process the increased data should not be a significant problem as the duration of the time window required at the high speeds is normally small.

Figure 9 shows the result using the measured track at the same speed. The period of the time window is increased in order to achieve a similar level of sensitivity/reliability for the detection of the peak value. This is due to the lack of high frequency components in the measured track data as explained earlier. In this case, a time shift of $46 \mathrm{~ms}$ is detected resulting in a truncation error of $0.55 \mathrm{~ms}$ ( or $0.65 \mathrm{~m} / \mathrm{s}$ ).

The noises or inaccuracies of the accelerometers needed for the speed measurement do not present a problem until the vehicle speed (and the excitation of track input) becomes very 


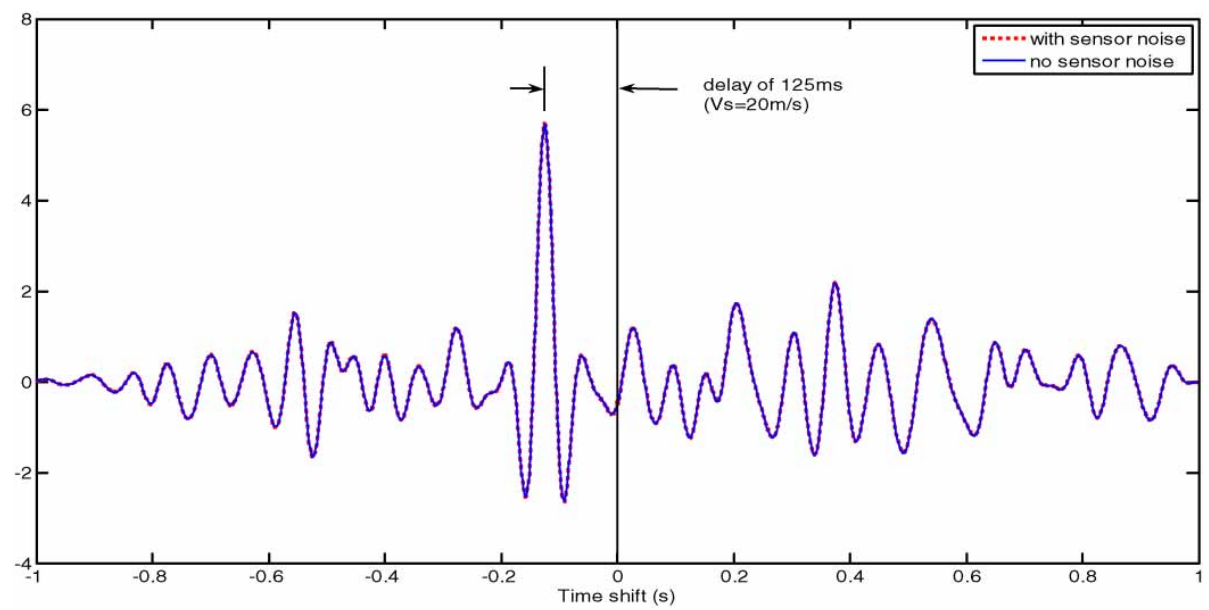

Figure 10. Cross-correlation of estimated track inputs (track 1, at $20 \mathrm{~m} / \mathrm{s}$ ).

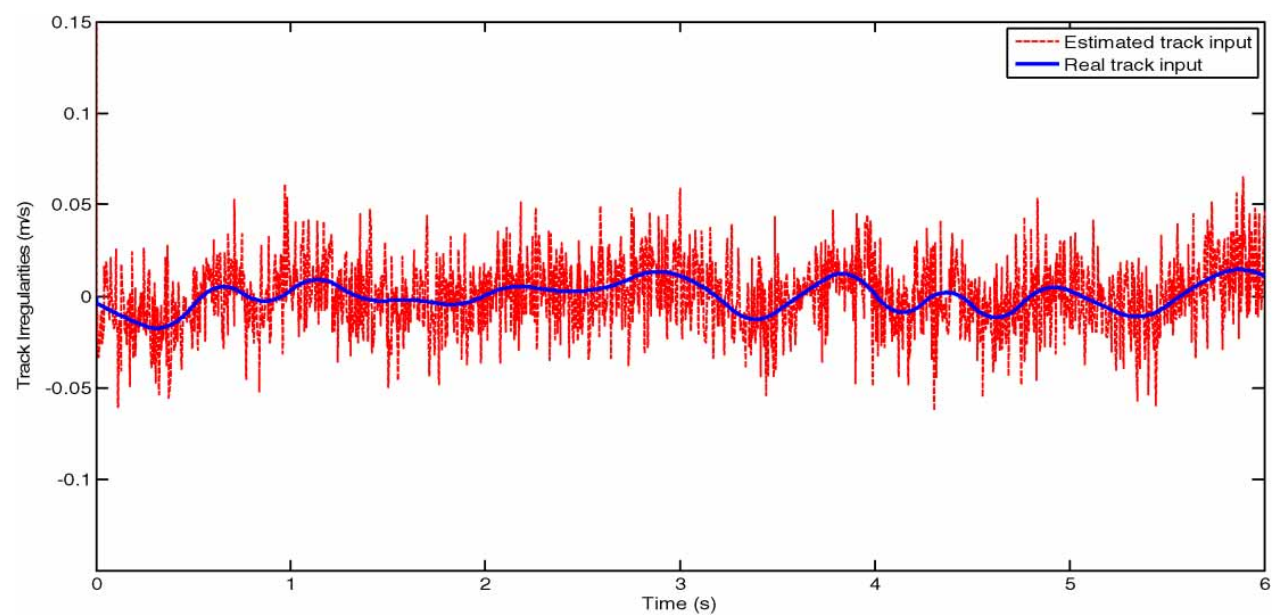

Figure 11. Estimated track irregularities - velocity (track 1, at $2 \mathrm{~m} / \mathrm{s}$ ).

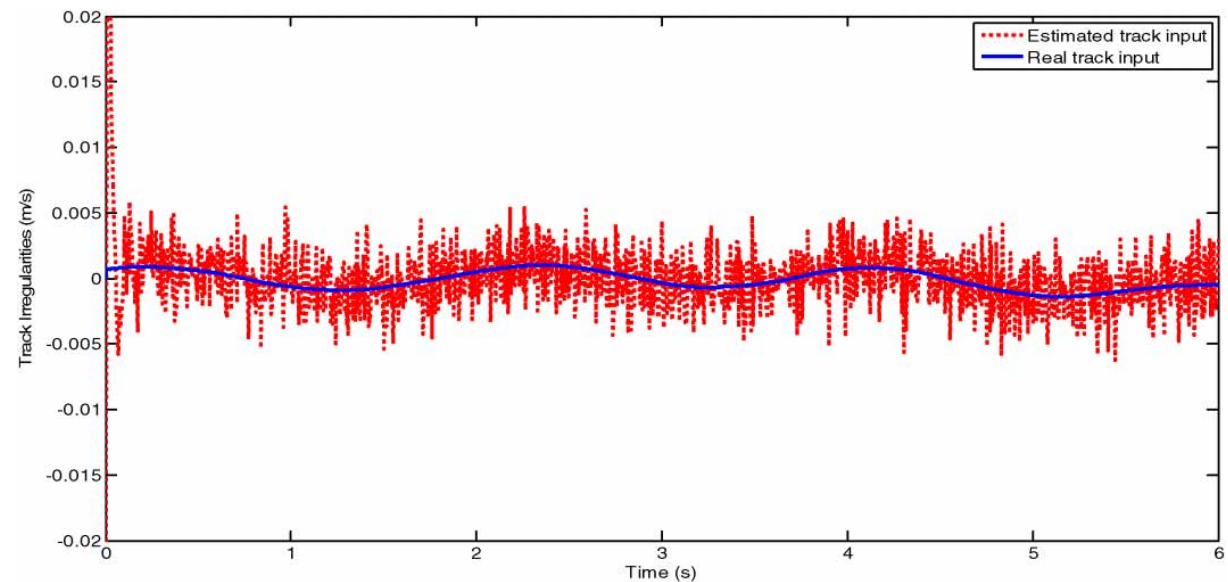

Figure 12. Estimated track irregularities - velocity (track 2, at $2 \mathrm{~m} / \mathrm{s}$ ). 
low. At the speed of $20 \mathrm{~m} / \mathrm{s}(72 \mathrm{~km} / \mathrm{h})$, there is no obvious effect at all as shown in Figure 10 where a sensor noise of $1 \%$ of the maximum measurement range is added in the simulation.

Even at very low speed, the proposed method shows an excellent robustness against external interference. Figures 11 and 12 indicate that, at the speed of $2 \mathrm{~m} / \mathrm{s}$, the sensor noises become much more dominant in the estimated track signals than the actual data for both the simulated and the measured inputs. However, the effect on detecting the time delay is negligible as demonstrated in Figures 13 and 14. For the track data 1, the measured vehicle speed is $2.0032 \mathrm{~m} / \mathrm{s}$ and $1.995 \mathrm{~m} / \mathrm{s}$ with and without the noises, respectively. For the track data 2, the detected speed is $2.024 \mathrm{~m} / \mathrm{s}$ and $1.995 \mathrm{~m} / \mathrm{s}$ with and without the noises, respectively. This is because the sensor noises do not cause correlations with the specific time shift that coincides with that between the two input signals. The only effect is at the zero time shift as indicated in the figures. Note that, at the very low speed, a much increased time window is needed that

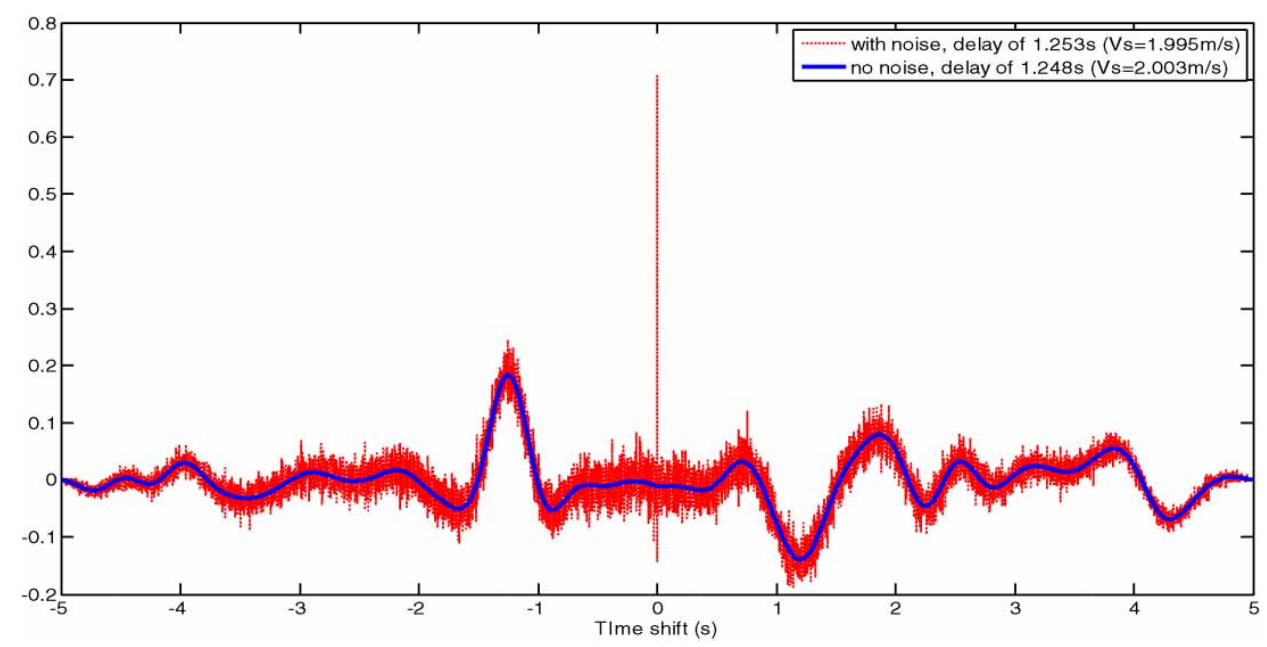

Figure 13. Cross-correlation of estimated track inputs (track 1, at $2 \mathrm{~m} / \mathrm{s}$ ).

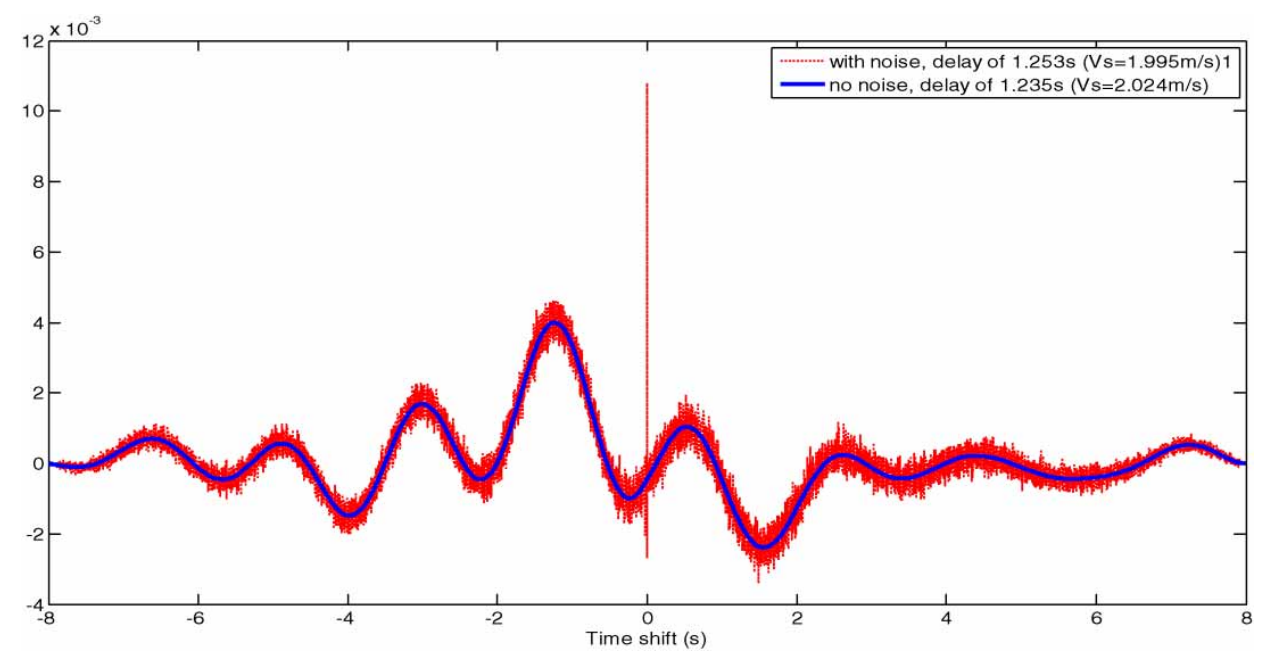

Figure 14. Cross-correlation of estimated track inputs (track 2, at $2 \mathrm{~m} / \mathrm{s}$ ). 
causes an undesirable update delay of several seconds for the speed detection and some form of compensation for the delay would be needed to overcome the problem.

The design of the filters for the estimation of the track inputs requires some knowledge of the vehicle parameters, and any inaccuracy will obviously have an impact on the outcome. Figure 15 compares the actual track data with the estimated ones from three different filter settings - the first with the original gains, the second with the damping ratio of the primary suspension reduced to $80 \%$ and the third with damping increased by $50 \%$. The first filter provides the closet estimation of the track input. The second filter produces an estimation substantial by greater than the actual track data, whereas output from the third filter is smaller than the track data.

The differences in the estimation are also reflected in the computed cross-correlations as shown in Figure 16. The time shift of the peak value in all the three cases remains the same,

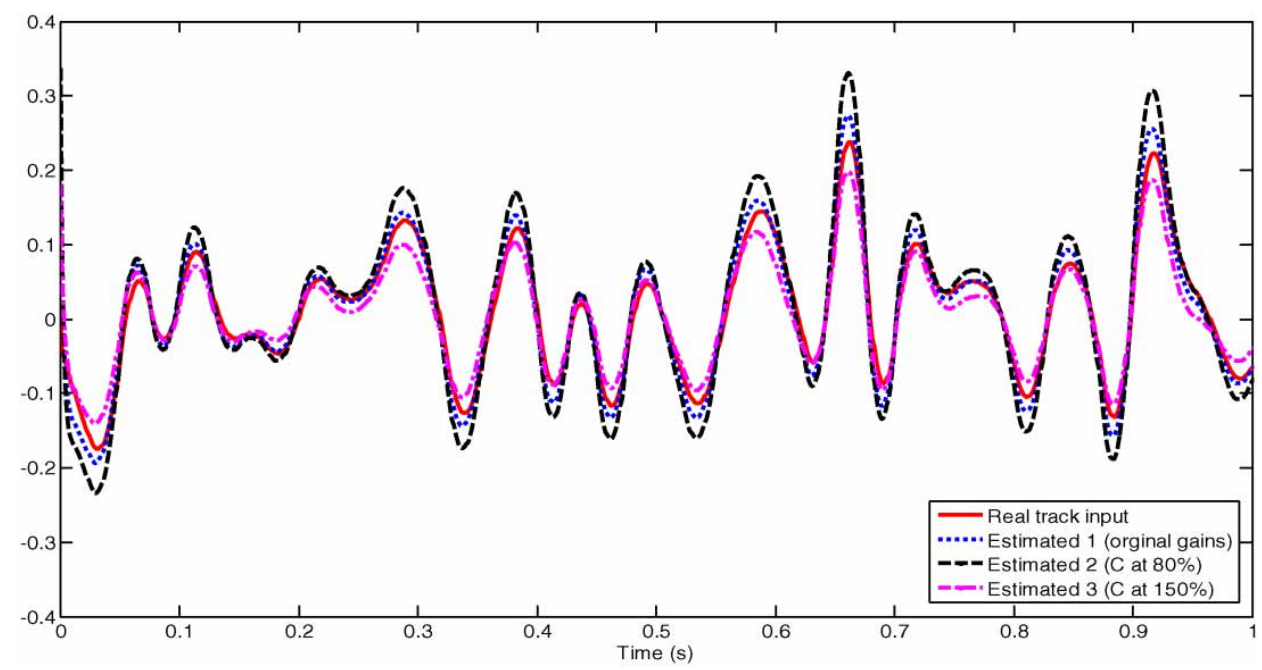

Figure 15. Estimated track irregularities - velocity (track 1, at $2 \mathrm{~m} / \mathrm{s}$ ).

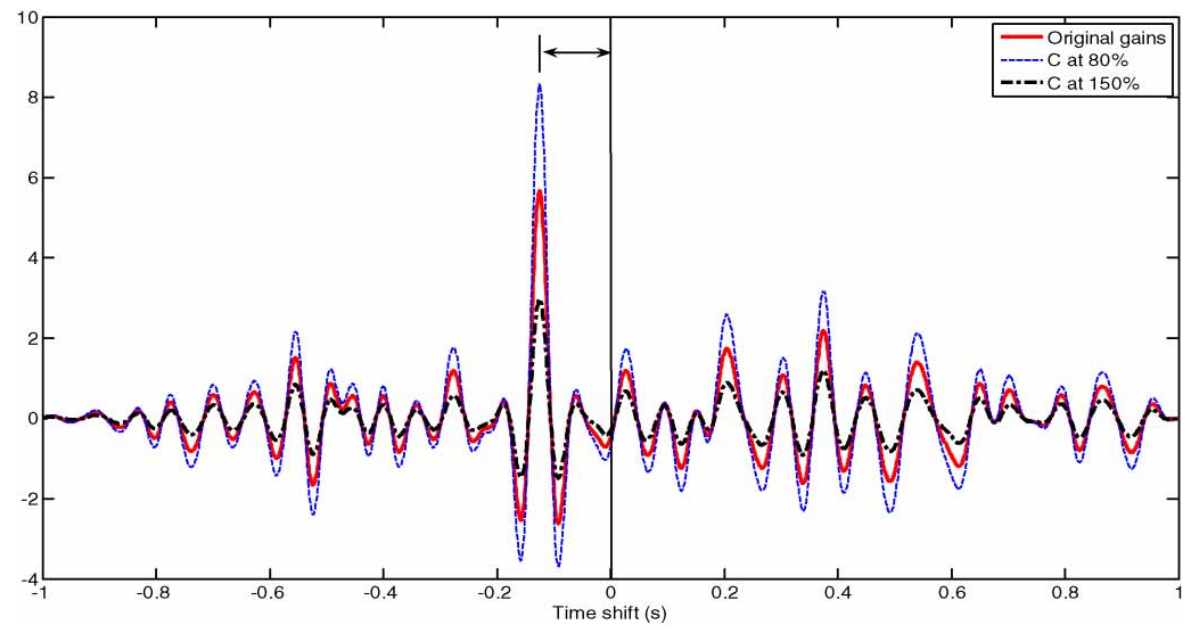

Figure 16. Cross-correlation of estimated inputs using different filters (track 1, at $20 \mathrm{~m} / \mathrm{s}$ ). 


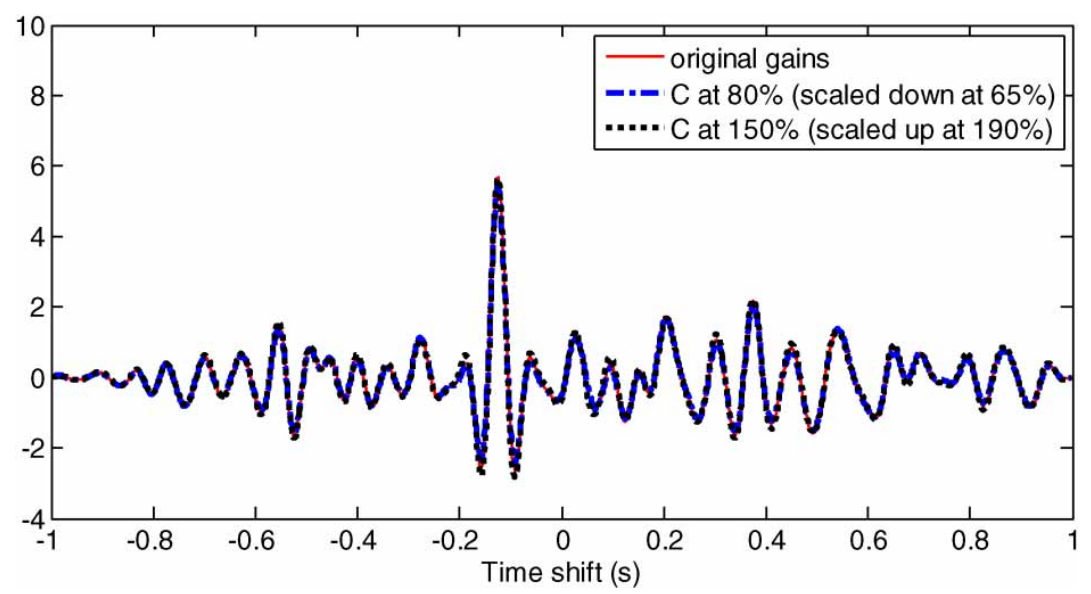

Figure 17. Cross-correlation of estimated inputs - re-scaled (track 1, at $20 \mathrm{~m} / \mathrm{s}$ ).

although the plot appears to give an impression that the sensitivity of detecting the time shift varies with the filter settings. However, a re-plot of the cross-correlations by scaling two of the results (by factors 0.65 and 1.9, respectively) reveals that, in relative terms between the peak and the other values, there is hardly any difference between the filters as demonstrated in Figure 17. This high level of robustness is possible because any imperfect information in the vehicle parameters affects the estimated outputs at both the wheelsets of bogie in exactly the same way in both magnitude and frequency which presents little influence on the time delay between the two channels.

\section{Conclusions}

The measurement of vehicle ground speed is very important for safe and reliable operations of a railway network. This paper has presented an indirect method that requires only the measurement of bogie vibrations using robust inertia sensors. Unlike the conventional encoderbased techniques, the proposed method will not be affected by wheel slip/slide conditions in traction or braking which is particularly problematic in poor contact conditions.

It has been demonstrated in the study that the new method may be used to provide an accurate measurement across a wide range of vehicle speed as it appears to work effectively even when the level of vibration is very low at extremely low speeds. The method has been shown to be insensitive to sensor noises and also robust against uncertainties or changes in the bogie parameters.

A reliable detection of the time shift of the track input at the two wheelsets of a bogie can be achieved by selecting a sensible time window to provide sufficient number of sampled data for the computation of cross-correlations. When a vehicle changes speed during acceleration or deceleration, a long time window (as required at very low speeds) would potentially cause a delay in the measurement which is the main limitation of the proposed method. A separate study is ongoing to develop a solution to compensate the problem.

As part of the study, this paper has also demonstrated that the track irregularities may be derived from bogie-based inertia sensor measurements by using fairly simple filtering techniques which would offer an easy and low cost alternative for the measurement and monitoring of track misalignment. 


\section{References}

[1] N. Kumagai, S. Uchida, I. Hasegawa, and K. Watanabe, Wheel slip rate control using synchronised speed pulse computing, International Conference on Computers in Railways, Bologne, Italy, 2000, pp. 623-632.

[2] R. Badmann, Measuring vehicle ground speed with a radar sensor, Sensors 13(12) (1996), pp. 30-31.

[3] T. Engelberg and F. Mesch, Eddy current sensor system for non-contact speed and distance measurement of rail vehicles, 7th International Conference on Computers in Railways, 2000, pp. 1261-1270.

[4] J. Bohmann, H. Meyr, and G. Spies, A digital signal processor for high precision non-contact speed measurement of rail guided vehicles, IEEE Vehicular Technology Conference, 1982, pp. 454-462.

[5] A. Harvey and H. Cohen, Vehicle speed measurement using an imaging method, IECON, 1991, pp. 1730-1733.

[6] P.I. Ostromenskij, Device for measurement of motion speed of railway transport facility, Russia patent RU2110803, 1998.

[7] T.X. Mei and H. Li, Measurement of vehicle ground speed using bogie based inertial sensors, IMechE Proc, Part F - Rail and Rapid Transit, 222 (2008), in press.

[8] T.X. Mei, H. Li, and R.M. Goodall, Kalman filtering applied to actively controlled railway vehicle suspensions, Trans. Instit. Measur. Contr. 23(3) (2001), pp. 163-181. 